\title{
Management of a Late-Term Hiatal Hernia with Intrathoracic Pouch Migration After Roux-en-Y Gastric Bypass
}

\author{
Theodoros Thomopoulos $^{1}$ (D) Maurice FitzGerald ${ }^{1} \cdot$ Styliani Mantziari ${ }^{1,2} \cdot$ Nicolas Demartines $^{1,2} \cdot$ Michel Suter $^{1,2,3}$
}

Received: 11 October 2021 / Revised: 30 December 2021 / Accepted: 30 December 2021 / Published online: 6 January 2022

(c) The Author(s) 2022

Keywords Hiatal hernia $\cdot$ Roux-en-y gastric bypass $\cdot$ Fundopli cation $\cdot$ Laparoscopic revision

\section{Introduction}

Hiatal hernia $(\mathrm{HH})$ with intrathoracic migration of the gastric pouch (IMGP) is a rare long-term complication of laparoscopic Roux-en-Y gastric bypass (LRYGB); however, its management remains poorly documented $[1,2]$. Several factors may contribute to the development of this entity. Rapid weight loss can lead to the relaxation of the phreno-esophageal ligament and enlargement of the hiatus [3] allowing for easier migration. Creation of a large gastric pouch could favor its migration because of the higher intragastric pressure [4]. Extended dissection of the cardia and

Theodoros Thomopoulos and Maurice FitzGerald contributed equally for the redaction of this paper.

\section{Key points}

- It is important for clinicians to consider intrathoracic migration of the gastric pouch as a differential diagnosis in patients complaining for reflux and dysphagia, with a history of previous LRYGB.

- A serious imaging work-up has to be undertaken pre-operatively, in order to assess the exact anatomic alterations of the LRYGB montage, as well as any associated esophageal dysmotility. - Laparoscopic-modified Nissen fundoplication after LRYGB, using the excluded stomach, is considered a technically demanding procedure. Nevertheless, it remains safe and efficient with minimal morbidity in experienced hands.

Theodoros Thomopoulos

theodoros.thomopoulos@chuv.ch

Maurice FitzGerald

maurice.fitzgerald@chuv.ch

Styliani Mantziari

styliani.mantziari@chuv.ch

Nicolas Demartines

demartines@chuv.ch

Michel Suter

michelsuter@netplus.ch the left crus during the LRYGB may disrupt normal attachments between the cardia and diaphragm and predispose to IMGP [5]. Finally, a small sliding HH may pass unperceived pre- or even intra-operatively, or intentionally not repaired during LRYGB, leading to progression of $\mathrm{HH}$ after bariatric surgery [6].

Patients with IMGP may present with epigastric/retrosternal pain, dysphagia, and regurgitations. In addition to the conventional imaging techniques used for the diagnosis of IMGP, such as CT scan and barium transit, the swallow magnetic resonance imaging (MRI) has been recently described as a new dynamic method to assess the anatomy of the gastric pouch, as well esophageal dysmotility after LRYGB, and could be of great use in the diagnostic armamentarium of swallowing difficulties after RYGB [7]. Various surgical techniques have been described in the literature to address HH and IMGP after LRYGB, yet these reports are sporadic and inconsistent. Among them, the hiatoplasty with the ligamentum teres hepatis [8], or a modified Nissen procedure using the fundus of the excluded stomach, may be valid options [9].

The purpose of the present work is to illustrate the main technical aspects of a laparoscopic-modified Nissen fundoplication with the excluded stomach to correct IMGP after RYBG.

1 Department of Visceral Surgery, University Hospital of Lausanne (CHUV), Rue du Bugnon 46, 1011 Lausanne, Vaud, Switzerland

2 Faculty of Biology and Medicine, University of Lausanne, Lausanne, Switzerland

3 Department of Surgery, Riviera-Chablais Hospital, Rennaz, Switzerland 


\section{Methods}

This video shows the case of a 54-year-old female patient, with an ante-colic LRYGB performed 14 years earlier in our institution for an initial BMI of $46 \mathrm{~kg} / \mathrm{m}^{2}$. The patient lost $45 \mathrm{~kg}$ during the first 2 years after surgery but regained almost $20 \mathrm{~kg}$ over the last 5 years with a current BMI of $38 \mathrm{~kg} / \mathrm{m}^{2}$. She presented with epigastric pain, reflux, and dysphagia to solid food with occasional postprandial vomiting. Although upper endoscopy did not reveal any anatomical anomaly, a barium swallow and a thoraco-abdominal CT scan showed herniation of the gastric pouch with an associated kinking effect at the level of the hiatus. Surgical management of the IMGP was therefore proposed.

\section{Results}

The laparoscopic exploration, lysis of adhesions, reduction of the $\mathrm{HH}$, posterior crural repair, and the modified Nissen fundoplication with the gastric remnant are demonstrated step-by-step in this video. The postoperative course was uneventful and she was discharged on POD3. At 6-month follow-up, she no longer had any abdominal pain and gastric reflux, and food tolerance was excellent.

\section{Conclusion}

IMGP is a well-known but rare complication of LRYGB, and bariatric surgeons need to be familiar with its management. In case of symptoms suggesting IMGP, a thorough pre-operative work-up is essential before proceeding to surgery. Surgical correction of IMGP with a modified Nissen fundoplication using the excluded stomach is a technically demanding procedure, but in experienced hands, it can be safely performed with minimal morbidity.

Supplementary Information The online version contains supplementary material available at https://doi.org/10.1007/s11695-021-05881-1.

Funding Open access funding provided by University of Lausanne.

\section{Declarations}

Human and Animal Rights All procedures performed were in accordance with the ethical standards of the institutional and/or national research committee and with the 1964 Helsinki Declaration and its later amendments or comparable ethical standards.
Informed consent was obtained from the individual included in the study to use the media from the surgery for educational purposes.

Competing Interests The authors declare no competing interests.

Open Access This article is licensed under a Creative Commons Attribution 4.0 International License, which permits use, sharing, adaptation, distribution and reproduction in any medium or format, as long as you give appropriate credit to the original author(s) and the source, provide a link to the Creative Commons licence, and indicate if changes were made. The images or other third party material in this article are included in the article's Creative Commons licence, unless indicated otherwise in a credit line to the material. If material is not included in the article's Creative Commons licence and your intended use is not permitted by statutory regulation or exceeds the permitted use, you will need to obtain permission directly from the copyright holder. To view a copy of this licence, visit http://creativecommons.org/licenses/by/4.0/.

\section{References}

1. Iannelli A, Kassir R, Schneck AS, et al. Hiatal hernia of the Roux-en-Y gastric bypass pouch 8 years after surgery. Obes Surg. 2014;24:1494-6.

2. Clapp B, Vo LU, Lodeiro C, et al. Late-term hiatal hernia after gastric bypass: an emerging problem. Surg Obes Relat Dis. 2020;16:471-5.

3. Sahle GP, Birch DW, Arya M, et al. Managing complications associated with laparoscopic Roux-en-Y gastric bypass for morbid obesity. Can J Surg. 2012;55:329-36.

4. Pandolfino JE, El-Serag HB, Zhang Q, et al. Obesity: a challenge to esophagogastric junction integrity. Gastroenterology. 2006;30:639-49.

5. Santonicola A, Angrisani L, Cutolo P, et al. The effect of laparoscopic sleeve gastrectomy with or without hiatal hernia repair on gastroesophageal reflux disease in obese patients. Surg Obes Relat Dis. 2014;10:250-5.

6. Docimo S Jr, Rahmana U, Bates A, et al. Concomitant hiatal hernia repair is more common in laparoscopic sleeve gastrectomy than during laparoscopic Roux-en-Y gastric bypass: an analysis of 130.772 cases. Obes Surg. 2019;29(2):744-6.

7. Felsenreich DM, Arnoldner MA, Langer FB, et al. Swallow magnetic resonance imaging compared to 3D-computed tomography for pouch assessment and hiatal hernias after Roux-en-Y gastric bypass. Obes Surg. 2020;30(11):4192-7.

8. Runkel A, Scheffel O, Marjanovic G, et al. Augmentation of hiatal repair with the ligamentum teres hepatis for intrathoracic gastric migration after bariatric surgery. Obes Surg. 2021;31(4):1422-30.

9. Kawahara NT, Alster C, Maluf-Filho F, et al. Modified Nissen fundoplication: laparoscopic anti-reflux surgery after Roux-en-Y gastric bypass for obesity. Clinics. 2012;67(5):531-3.

Publisher's Note Springer Nature remains neutral with regard to jurisdictional claims in published maps and institutional affiliations. 